ADHD is hypothesized on the basis of cerebral blood flow and EEG studies, and MRI findings. The concept develops from the function of the frontal lobe as an inhibitor of excessive motor activity, and children with ADHD having disinhibited motor activity. The calming effect of methylphenidate stems from its stimulatory effect on the frontal lobe causing motor inhibition. (Niedermeyer E, Naidu SB. Attention-deficit hyperactivity disorder (ADHD) and frontal-motor cortex disconnection. Clin Electroencephalogr July 1997;28:130136). (Reprints: Ernst Niedermeyer MD, Johns Hopkins Hospital, Room 2-147, Meyer Building, $600 \mathrm{~N}$ Wolfe Street, Baltimore, MD 21287).

COMMENT. The importance of the frontal lobe in relation to hyperactive behavior was demonstrated more than 50 years ago in animal studies by Langworthy and Richter (1939), and later by Livingston, Fulton and colleagues (1948), and Mettler (1967). Two types of hyperactivity were distinguished: overreactivity, an external stimulation or distractibility caused by frontal lobe injury, and essential overactivity, a disinhibitive hyperkinesia due to striatal lesions and release from fronto-cortical-reticular inhibition of ascending systems. (See Progress in Pediatric Neurology III, Ed. Millichap JG, PNB Publ, pp195-265, for further articles and comments on the neurobiologic organic theory of ADHD). Since the report of 3 children with a temporal lobe arachnoid cyst TLAC/ADHD syndrome (Millichap JG. Neurology May 1997;48:1435-1439),

three further cases have been uncovered. Different sites and degrees of damage in the cortical striatal circuit might account for the heterogeneous nature of ADHD.

\title{
HIPPOCAMPAL SCLEROSIS AND COGNITIVE DYSFUNCTION
}

Deficits of cognitive function, including language development and learning of complex social skills, typical of mental deficiency and autism, are reported in 4 children with bilateral hippocampal sclerosis and epilepsy followed at Duke University Medical Center, Durham, NC. MRI showed increased signal on T2-weighted images and $25 \%$ loss of volume of the hippocampi, pictures consistent with sclerosis. An early normal development was interrupted by episodes of status epilepticus, followed by failure of language development and social deficits. Control of seizures was not accompanied by improved social/adaptive skills. The hippocampus is essential for learning of language and social development in infancy. (DeLong GR, Heinz ER. The clinical syndrome of early-life bilateral hippocampal sclerosis. Ann Neurol July 1997;42:11-17). (Respond: Dr DeLong, Division of Pediatric Neurology, Box 3936, Duke University Medical Center, Durham, NC 27710).

COMMENT. Bilateral hippocampal sclerosis in infancy may result in failure of language development and deficits in behavior and social learning consistent with mental retardation and autism. Normal acquisition of language and learning is not significantly affected by unilateral damage to the hippocampus. Neuronal loss in the hippocampi during prolonged seizures has been linked to excessive release of glutamate.

\section{RIGHT BRAIN DOMINANCE IN INFANCY}

The functional development of the left and right cerebral hemispheres in 39 normal children, aged 18 days to 19 years, was studied by measurements of cerebral blood flow at rest, using single photon emission computed tomography (SPECT), at the Neuropediatric Department, Hospital Saint Vincent de Paul, Paris, France. Functional brain activity and blood flow were greater in 
the right hemisphere in children between 1 and 3 years of age, whereas the left hemisphere was functionally dominant at 4 years and later. The change from right to left asymmetry was due to a shift in activity of the posterior associative area. This shift of asymmetry appeared to be functionally related to the visuospatial abilities subserved by the right hemisphere in the first year followed by emergence of language abilities at 3 years of age, a left hemisphere function. (Chiron C, Jambaque I, Nabbout R et al. The right brain hemisphere is dominant in human infants. Brain June 1997;120:1057-1064). (Respond: Dr Catherine Chiron, Service de Neuropediatrie, Hopital Saint Vincent de Paul, 82 avenue Denfert Rochereau, 75674 Paris cedex 14, France).

COMMENT. Right hemisphere cerebral blood flow (CBF) is higher than left at 1 to 3 years of age, whereas left $\mathrm{CBF}$ is higher in older children. Right hemisphere of infants is functionally dominant whereas the left becomes dominant after 3 years, when language develops. These significant CBF asymmetries are detected in sensorimotor cortex, Broca's area and the posterior associative regions which serve handedness and language.

\section{RETT SYNDROME}

\section{EARLY BRAIN GROWTH IN RETT SYNDROME}

Neuropathological and neurochemical studies supporting the concept of Rett syndrome (RS) as a neurodevelopmental disorder are reviewed from the Kennedy Krieger Institute and Johns Hopkins Hospital, Baltimore, MD. Prevalence in females is $1: 10,000$ to $1: 22,000$. Incomplete, forme fruste, cases are common. Twin data support a genetic basis but its nature is undetermined and no biological marker has been defined. Reduction in velocity of head growth begins at 2 to 4 months of age. Brain weight and size are reduced by 12 to $34 \%$ compared to age-matched controls in autopsy studies, but without evidence for progressive decrease in brain weight with age. Immunological studies suggest that certain pyramidal neurons are preferentially affected. Low levels of dopamine receptors and transporters are associated with decreased dopamine levels in the neocortex and basal ganglia. Choline acetyltransferase is reduced in the neocortex, hippocampus, putamen, and thalamus. Abnormal neurotransmitter systems can account for the clinical manifestations of RS. The early rapid evolution of symptoms followed by a static course suggest a neurodevelopmental disorder. (Naidu S. Rett syndrome: a disorder affecting early brain growth. Ann Neurol July 1997;42:3-10). (Respond: Dr SakkuBai Naidu, Kennedy Krieger Institute, \#500 707 N Broadway, Baltimore, MD 21205).

COMMENT. Rett syndrome as a specific disease still eludes definition. Its static course after age five years is at variance with most genetic neurodegenerative disorders, and an abnormality in neurodevelopment appears more likely. My colleague Dr John Wilson at Great Ormond Street Hospital, London, suggests that the age-specific decelerating head growth in RS may be an apoptotic phenomenon.(In: Progress in Pediatric Neurology III, PNB Publ, 1997;p567).

A prevalence rate for RS of 2.17 per 10,000 girls is reported in a Norwegian study. (Skjeldal OH et al. Brain Dev June 1997;19:258-261). This higher than usual rate was associated with clustering in certain restricted geographical areas, a finding important in identifying a possible genetic basis 